\title{
Intra-vascular leiomyoma of the popliteal vein
}

\author{
R.J. Grimer and G.R. Armstrong
}

The Birmingham Bone Tumour Treatment Centre, Royal Orthopaedic Hospital, Bristol Road South, Northfield, Birmingham B31 2AP, UK.

\begin{abstract}
Summary: A large mass in the popliteal fossa was found to be a leiomyoma of the popliteal vein with portions of tumour both inside and outside the vein. This is only the second recorded case of a benign smooth muscle tumour of a peripheral vein.
\end{abstract}

\section{Introduction}

Leiomyomas are benign smooth muscle tumours which occur principally in the uterus. In other sites they are usually considered to have arisen from smooth muscle and in some instances may arise from the smooth muscle wall of a blood vessel. Leiomyomas actually arising within veins are rare and most reported cases have been in relationship to the inferior vena cava or have spread up from the uterus.

\section{Case report}

A 45 year old man presented with a two year history of discomfort behind the right knee. He had noticed the presence of a mass which had slowly increased in size over this time. He commented that he occasionally developed pins and needles of the right foot if he sat for long in a firm chair but at no time did he have any swelling of the leg.

Examination revealed a firm mobile swelling in the proximal popliteal fossa which did not appear to be deeply fixed. The knee had a full range of movement and there was no neurological or vascular deficit. X-rays revealed a soft tissue swelling in the popliteal fossa.

The mass was biopsied and histology revealed hyaline fibrous tissue only. The mass was subsequently excised through a curved posterior approach and was found to be $8 \times 5 \mathrm{~cm}$ long, well encapsulated with a shiny surface. The sciatic nerve was easily reflected off its surface but the mass was found to be tethered deeply to the wall of the popliteal vein. On further inspection a small nodule of tumour was clearly seen inside the vein. A $2 \mathrm{~cm}$

Correspondence: R.J. Grimer, F.R.C.S., F.R.C.S.Ed(Orth). Accepted: 10 September 1987 length of vein containing the tumour was resected and an end-to-end anastamosis carried out. The patient made an uneventful postoperative recovery.

On bisecting the tumour through the nodule it became obvious that the two portions of tumour on either side of the vein were in continuity with each other and the main portion of the tumour had a rubbery texture and a whorled appearance much like a 'fibroid'. Histological examination of the whole of the specimen showed that for the most part the tumour comprised hyalinized fibrous tissue. However, the area protruding through the vein wall and immediately below it was moderately cellular comprising plump spindle shaped cells with uniform cigar shaped nuclei. Using a Masson trichrome stain the cells had pink cytoplasm identifying them as smooth muscle cells (Figure 1). Small bundles of similar cells were seen scattered throughout the hyalinized areas. There was no evidence of cellular atypia and no mitotic figures were encountered. The lesion was therefore diagnosed as a benign smooth muscle tumour or leiomyoma. Although the tumour passed completely through the vein wall no direct origin in the smooth muscle of the vein could be identified. The abundance of hyalinized fibrous tissue is probably a feature of 'ageing' in this particular tumour, although it could have arisen as a result of repetitive trauma (e.g. sitting on a chair) although in that case we would have expected to have observed the stigmata of trauma such as haemosiderin occurring secondary to haemorrhage within the tumour.

\section{Discussion}

Smooth muscle tumours of veins are rare entities and are usually leiomyosarcomas. Kevorkian and Cento $^{1}$ reviewed published reports of smooth

(C) The Fellowship of Postgraduate Medicine, 1988 


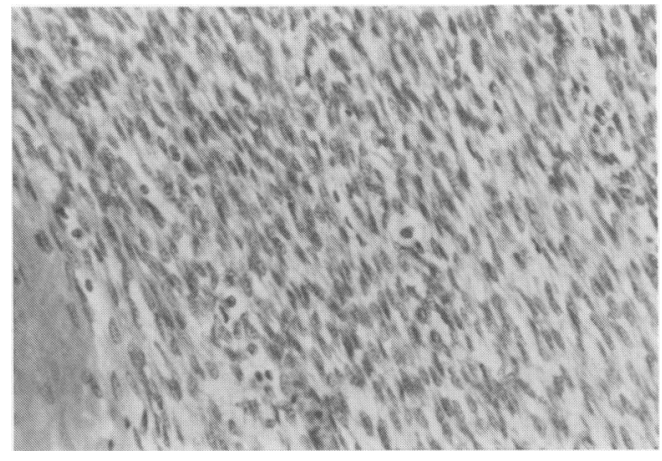

Figure 1 Cellular element of the tumour demonstrating spindle cell with cigar-shaped nuclei typical of a benign smooth muscle tumour (Masson trichrome stain $\times 200$ ).

muscle tumours of vein and arteries and were unable to find a single benign tumour. In 1977 Karmody et al. ${ }^{2}$ reported a single case of leiomyoma of the external iliac vein and commented that almost all tumours greater than $5 \mathrm{~cm}$ in size had an aggressive malignant potential whilst those smaller than that were of lower grade malignancy. Fischer et $a .^{3}{ }^{3}$ reviewed primary smooth muscle tumours of venous origin and reported on one leiomyoma of the jugular vein, commenting that most other tumours were malignant. The malignant potential of smooth muscle tumours is notoriously difficult to assess and there is probably a biological continuum from benign to malignant. Although size, cellularity, atypia and necrosis are all suggestive of malignancy the only reliable predictor of malignancy is the mitotic rate. For smooth muscle tumours outside the uterus an arbitrary figure of 5 mitoses per 10 high power fields is indicative of malignancy. ${ }^{4}$ The tumour in our case is benign by all histological criteria except for size. Follow-up will, however, be required as the only absolute criterion for malignancy is metastatic disease.

Most smooth muscle tumours occur more centrally in the body and the occurrence of one peripherally has been sufficiently rare for several authors to comment upon. Goodman and Briggs ${ }^{5}$ reported a leiomyoma of the thigh in a 5 year old which they presumed to have arisen from smooth muscle cells of a blood vessel's wall. Bulmer ${ }^{6}$ reported on 10 cases of smooth muscle tumours of the limbs of which only two were not in the superficial tissues and neither of these arose from blood vessels.

We have not found a single case which demonstrates a similar 'dumb-bell' type of tumour in a peripheral vein. It is well recognized $^{7}$ that leiomyomas of the uterus can spread into veins and produce the condition of intravenous leiomyomatosis and benign smooth muscle tumours of the inferior vena cava have been reported to produce a similar effect. ${ }^{8}$

This particular case is rare in that it is not only a benign smooth muscle tumour, presumably arising from a vein wall, but also that it should have components both within and outside the vessel necessitating resection of part of the vessel to remove the tumour.

\section{Acknowledgements}

We would like to thank Mr R.S. Sneath for allowing us to report this case, $\mathrm{Mr} \mathrm{R}$. Sage for his surgical assistance and Mrs H. Sugrue for her secretarial help.

\section{References}

1. Kevorkian, J. \& Cento, D.P. Leiomyosarcoma of large arteries and veins. Surgery 1973, 73: 390-400.

2. Karmody, A.M., Zaman, S.N. \& Sarfeh, J.I. Leiomyoma of external iliac vein. NY State J Med 1977, 77: 2279-2281.

3. Fischer, M.G., Gelb, A.M., Nussbaum, M., Haveson, S. \& Ghali, V. Primary smooth muscle tumors of venous origin. Ann Surg 1982, 196: 720-724.

4. Enzinger, F.M. \& Weiss, S.W. In: Soft Tissue Tumours. C.V. Mosby, London, 1983, pp 298-315.
5. Goodman, A.H. \& Briggs, R.C. Deep leiomyoma of an extremity. J Bone Joint Surg (Am), 47: 529-532.

6. Bulmer, J.H. Smooth muscle tumours of the limbs. $J$ Bone Joint Surg (Br) 1967, 49: 52-58.

7. Norris, H.J. \& Parmley, T. Mesenchymal tumours of the uterus. V. Intravenous leiomyomatosis. Cancer 1975, 36: 2164-2178.

8. Wray, R.C. \& Dawkins, H. Primary smooth muscle tumors of the inferior vena cava. Ann Surg 1971, 174: 1009-1018. 